\title{
Intervista al Prof. Gherardo Buccianti, pioniere dei nefrologi ecografisti
}

\author{
Antonio Granata per conto del Gruppo di Studio di Ultrasonologia della Società Italiana di Nefrologia \\ Unità Unità Operativa Complessa di Nefrologia e Dialisi, Ospedale “S. Giovanni di Dio", Agrigento
}

Interview with Prof. Gherardo Buccianti pioneer of the ultrasonography for nephrologists B-mode ultrasonography has always played a fundamental role in the field of renal disease. In Italy, the creation of the Ultrasound Study Group of the Italian Nephrology Society in 1991 represented an important step in the history of Italian ultrasound.

Since then, the number of nephrologists interested in ultrasonography has continuously increased, and the Ultrasound Study Group is now the Ultrasound Study Group in Nephrology. Nephrologists have indeed improved their ability not only in the field of renal disease, but also in heart and lung disease.

Professor Buccianti, one of the creators of the Ultrasound Study Group, will describe the most important phases of Italian Ultrasonography in Nephrology, on the occasion of the 25th Anniversary of the Study Group.

Keywords: Ultrasound, Nephrology, History

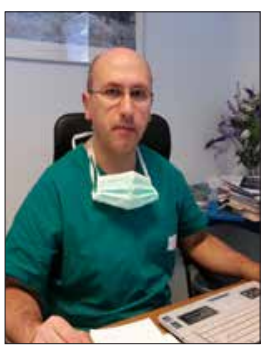

Antonio Granata
Professore (Fig. 1), Lei è stato cofondatore del GdS di ecografia renale della Società Italiana di Nefrologia (GSER/SIN) in un momento di grande fermento e crescita per la nefrologia italiana. Tra i suoi fondatori (tra cui ricordiamo con piacere il Prof. Nicola Di Paolo), un piccolo, volitivo ed entusiasta gruppo di nefrologi interessati ad approfondire l'uso degli ultrasuoni nello studio delle malattie renali. Dopo circa 25 anni, era il 1991, la validità dell'iniziativa è più che mai attuale. Da allora il numero dei "nefrologi ecografisti" ha subito una continua crescita (oggi siamo circa 400), così come i reparti e gli ambulatori di nefrologia. Nonostante le fasi iniziali siano state caratterizzate da rapporti "difficili" con i radiologi, oggi i nefrologi sono riusciti ad affermarsi come ecografisti di "qualità" e sono parte integrante delle più importanti Società specialistiche

Accepted: March 13, 2015

Published online: April 14, 2015

Indirizzo per la corrispondenza:

Dr. Antonio Granata

Via F. Paradiso 78/a

95024 Acireale (CT)

antonio.granata4@tin.it nazionali (SIUMB, SIEUN) e internazionali (EFSUMB). Le chiedo, al di là delle motivazioni ufficiali riportate nell'atto costitutivo del GdS, quali siano state le reali spinte che hanno indotto Lei e gli altri "fantastici" quattro (oltre Nicola Di Paolo, Franco Petrarulo, Luigi Capotondo e Paolo Conti) a intraprendere un viaggio saturo di difficoltà ma caratterizzato anche da così tanti riscontri positivi; inoltre, chi è stato il motore propulsore di tale idea?

Grazie per la domanda e per i gentili commenti.

Ricordo il 7 Dicembre 1991, festa di Sant'Ambrogio, come un giorno freddo e piovoso. Dopo una giornata di lavoro al Corso di Aggiornamento del Prof. D’Amico, Nicola Di Paolo, grande Nefrologo e mio caro amico, aveva invitato tutti i nefrologi a presentarsi alle 18.30 presso la sala congressi dell'Hotel Hilton di Milano. Ancora una riunione? A quest'ora? Pensai. Ma a Nicola non si nega mai niente, perché dietro c'è sempre un grande progetto!

E così, nel corso di quella riunione, Nicola ci parlò dell'ecografia e in particolare di quella renale, della necessità di conoscerla meglio e di introdurre l'ecografo quale strumento utile nei nostri centri di nefrologia.

Quella stessa sera si costitui l'Assemblea dei Soci Fondatori e venne approvato lo statuto ed eletto il primo Comitato Scientifico (allora lo chiamavamo cosi), composto da Nicola Di Paolo (Siena)(Coordinatore), LionelloScarpioni(Piacenza)(Segretario), Paolo Conti (Sorgono), Franco Petrarulo (Bari), Luigi Capotondo (Siena) e Gherardo Buccianti (Milano) (Consiglieri). 


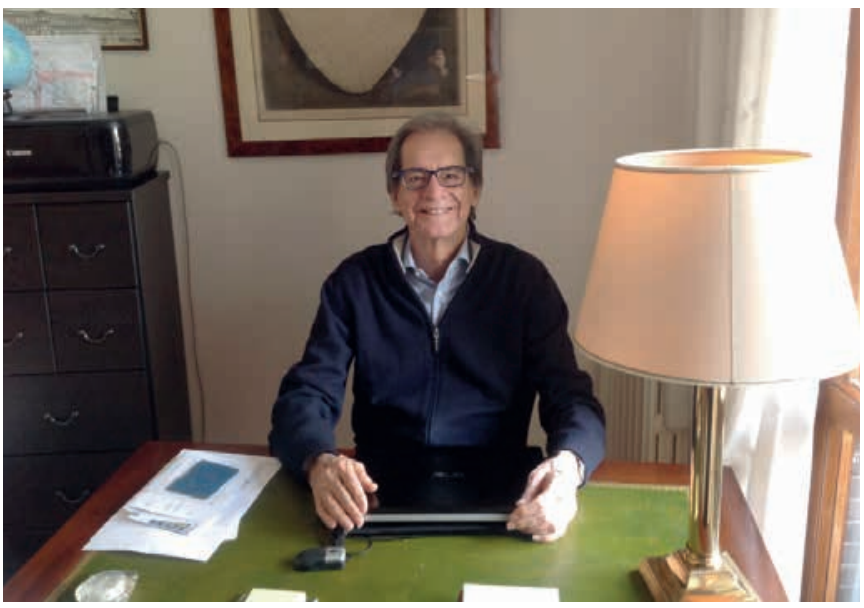

Fig. 1 - II Prof. Gherardo Buccianti come sempre in attività nel suo studio.

Ricordo, inoltre, l'entusiasmo di tutti nella pubblicazione sul Giornale di Tecniche Nefrologiche e Dialitiche degli atti del primo Convegno.

Il gruppo organizzò nel 1992 e nel 1993, presso l'Hotel Michelangelo di Milano, due riunioni sull'anatomia ecografica renale, sulla biopsia ecoassistita/guidata e sullo studio ecografico del rene trapiantato.

Nel 1993, al rinnovo del Comitato Scientifico, io venni nominato Coordinatore per il triennio 1994/1996 e come Consiglieri furono nominati Umberto Buoncristiani (Perugia), Fabrizio Dazzi (Pietrasanta) e Bruno Di Paolo (Chieti) e come Segretario Maria Zanazzi (Firenze). Quest'ultima svolse un lavoro stupendo effettuando la prima indagine nazionale sull'impiego dell'ecografia nei centri di nefrologia.

\section{Qual è l'episodio che, con particolare trepidazione, ricorda di quel periodo?}

In quel periodo a scopo didattico organizzammo numerosi Corsi/Convegni, come i precedenti presso l'Hotel Michelangelo nel 1994 e nel 1995, alcuni da me coordinati, in cui eseguimmo per la prima volta in tempo reale da un altro piano del centro congressi tutte le indagini ecografiche su pazienti affetti da patologia renale. Fu un grande successo e tutti i Colleghi, dai più giovani ai meno giovani, rimasero colpiti dalla semplicità e dalla velocità con la quale si riusciva a fare diagnosi.

Già nei Convegni del 1996-1997 avevamo avuto la possibilità di discutere dei principali argomenti inerenti la patologia nefrologica, dalle cisti renali congenite e acquisite ai tumori del rene, dalla patologia genitourinaria all'internistica fino all'interventistica. Proprio quando iniziavamo a padroneggiare le allora avanzate metodiche Doppler, si presentavano ulteriori sfide. Una di queste era rappresentata dalla comparsa

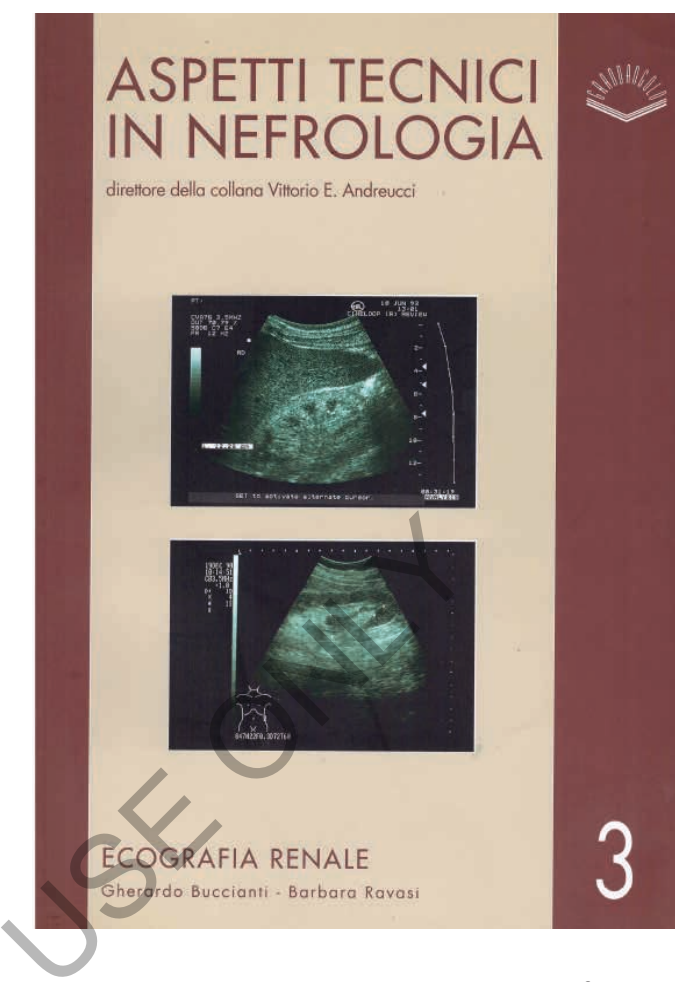

Fig. 2 - Frontespizio del I volume del libro di ecografia renale.

in commercio dei mezzi di contrasto di prima generazione. In quest'ultimo caso, non essendo preparati e non possedendo la tecnologia necessaria per fare fronte a questa nuova sfida, il tutto si tradusse in un flop.

Nel 1997 eravamo pronti e, spinti dall'instancabile Prof. V.E. Andreucci assieme a Barbara Ravasi (Servizio di Nefrologia e Dialisi, Ospedale Clinicizzato S. Donato Milanese), pubblicammo il primo volume (Fig. 2) sull'Ecografia Renale nella collana "Aspetti Tecnici in Nefrologia" allora diretta dal Prof. Andreucci.

Come in tutti i progetti, anche nel GdS di Ecografia Renale si è presentato un periodo di stasi. Intorno ai primi anni del 2000 si è verificato un certo distacco del GdS dalla realtà nefrologica: il Comitato Scientifico non riusciva più a suscitare l'interesse dei nefrologi (soprattutto dei più giovani) e alle riunioni e ai corsi partecipava un numero sempre più esiguo di Colleghi. Un particolare riconoscimento, a tal proposito, va a Fulvio Fiorini, ad Alessandro D'Amelio e a lei per aver rivitalizzato, con entusiasmo e lavoro, il gruppo di ecografia coinvolgendo attivamente tutti coloro che si erano proposti nel corso degli anni come cultori della materia, senza aver mai trovato uno spazio collaborativo in campo ecografico nefrologico. II GSER/SIN è stato, così, prepotentemente rilanciato e, da allora, è il più attivo e il più vitale dal punto di vista delle pubblicazioni scientifiche e dell'attività formativa fra i GdS della SIN. 


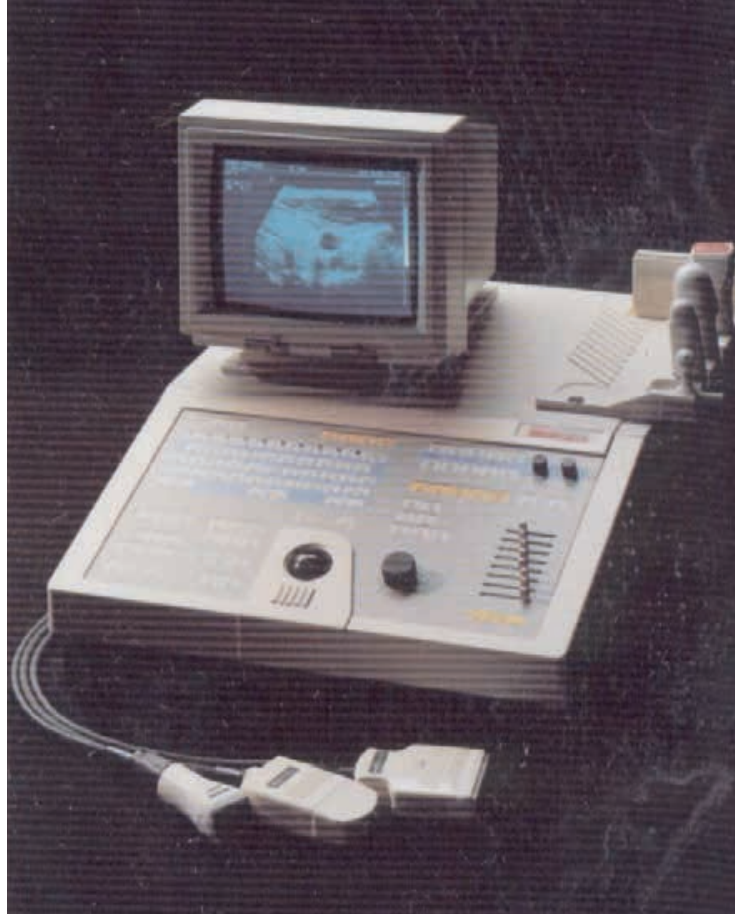

Fig. 3 - Ecografo analogico con trasduttori meccanici (Modello Esaote AU530, per gentile concessione del Dr. G. Riotino, Application UItrasound Esaote).

Qual era la tecnologia ecografica di cui disponevate in quel periodo?

L'ecografia ha mosso i suoi primi passi ed è entrata nella pratica clinica agli inizi degli anni'70, quando trovava impiego soprattutto in campo radiologico e cardiologico.

Ricordo che, verso la metà degli anni ' 80 , conobbi il Prof. Gasparini, radiologo del Policlinico di Milano passato poi al San Raffaele, entusiasta di questa nuova tecnica che, per la prima volta, sottraeva all'operatore e al paziente il rischio delle radiazioni: era quindi totalmente non invasiva.

La tecnologia era ovviamente diversa (Fig. 3), iniziava a farsi largo il concetto di real time, ma si trattava di apparecchi Compound, che impiegavano sonde meccaniche e non fornivano i migliori risultati: le immagini erano color seppia o tendenti al verde.

Devo infatti ammettere che era necessario avvalersi di un interprete per capire un'immagine spesso sfocata. Questa tecnica era tuttavia già un passo avanti rispetto alle altre e io prendevo atto sia dei suoi limiti che delle sue potenzialità.

Va ricordato ai più giovani che Lei ha iniziato l'attività di nefrologo negli anni '70 presso l'Istituto di Clinica Medica I del Policlinico di Milano per poi completarla come Direttore di UOC di Nefrologia e Dialisi e Capo Dipartimento di Medicina presso I'Ospedale San Gerardo di Monza passando per la docenza presso la Scuola di Specializzazione in Nefrologia dell'Università degli Studi di Milano. In quegli anni qual è stato l'aiuto principale che l'ecografia Le ha dato durante l'attività clinica, di ricerca e di docenza?

L'ecografia $B$-mode ha rivoluzionato la pratica clinica, basti pensare da un lato alla complessità, ai potenziali effetti collaterali e alla "scarsa" accuratezza diagnostica delle metodiche di imaging di quel tempo e, dall'altro, alla rapidità con la quale si riusciva a dare una "discreta" risposta al paziente e ai suoi familiari. Sicuramente l'aspetto più interessante della metodica va ricercato nella possibilità che ci ha dato sia di capire se il paziente fosse affetto da una nefropatia acuta, quindi potenzialmente reversibile, o da una nefropatia cronica che di eseguire con estrema sicurezza real time la biopsia renale o il cateterismo venoso centrale. Ricordo come se fosse ieri la mia prima biopsia renale, il tremore delle mani e l'emozione del momento che lasciavano il posto alla professionalità e al desiderio di dare una risposta al paziente, in altre parole di fare diagnosi. Sì, indubbiamente il regalo più bello che l'ecografia mi ha fatto e che spero faccia a tutti i Colleghi, specialmente ai giovani, è quello di fare direttamente DIAGNOSI, senza necessità di altre figure professionali.

Bisogna assolutamente non dimenticare il ruolo delle metodiche ultrasonografiche nel follow-up del rene trapiantato che, insieme ai dati clinici e bioumorali, consentono una significativa rapidità di intervento in corso di rigetto, nel posizionamento e nelle complicanze del catetere peritoneale, nel cateterismo venoso centrale e per via periferica, nella valutazione dello stato di idratazione, nello studio dei vasi pre e post confezionamento FAV e così via.

Nel corso di questi anni quali sono state le innovazioni tecnologiche che hanno principalmente destato il Suo interesse?

Sono sempre stato un fautore della necessità, per il nefrologo, di allargare le proprie competenze, non per fare un lavoro diverso, ma perché è ormai noto a tutti che egli è anche e soprattutto un buon internista che oggi deve confrontarsi con le patologie emergenti in campo vascolare e diabetologico. È, pertanto, doveroso un approfondimento delle competenze in campo ecocardiografico, come abbiamo iniziato a fare quest'anno al Corso di Ecografia Renale che si terrà a Brescia dal 16 al 20 Marzo 2015.

Sappiamo, infatti, che uno dei principali fattori che innesta le nuove dinamiche è costituito dall'ipertensione arteriosa, per cui la rigidità delle pareti vascolari che si sviluppa nei pazienti ipertesi e l'avanzamento dell'età costituiscono importanti fattori prognostici di morbilità e mortalità; per cui, ritengo molto utile la valutazione dell'Arterial Stiffness, che 
costituisce un recente e importante fattore di inquadramento della patologia.

Tra le innovazioni tecnologiche di sicuro interesse possiamo citare l'Elastosonografia, che fornisce informazioni sulla compattezza di un tessuto o di un nodulo che, sino a oggi, si potevano ottenere solo con la palpazione, per cui possiamo parlare di "palpazione elettronica", e l'Ecocontrastografia (CEUS), che ha il vantaggio di utilizzare agenti di contrasto che non comportano rischi e danni nel paziente nefropatico, aumentando le capacità del sangue di riflettere gli ultrasuoni mediante l'impiego di microbolle. Queste ultime si dissolvono dopo pochi minuti grazie all'azione del metabolismo epatico che ne elimina le pareti e dei polmoni, che eliminano il gas in esse contenuto. Per questo motivo, la CEUS trova grande applicazione sia nella diagnosi differenziale tra lesioni solide e cisti, specialmente quelle complicate, che tra tumori solidi e angiomiolipomi. Per rimarcare l'importanza dell'effettuare diagnosi minimizzando gli effetti sul paziente, basti pensare che i mezzi di contrasto tradizionali non potrebbero essere somministrati alla maggior parte dei soggetti affetti da insufficienza renale cronica che giungono alla nostra osservazione e, quando ciò avviene, per eseguire TAC o RMN, questi ultimi pagano spesso un prezzo elevato in termini di peggioramento della funzione renale.

Tuttavia, sul ruolo dell'ecocontrastografia in nefrologia, di cui Lei vanta un significativo numero di pubblicazioni, spetta a Lei, insieme agli altri Colleghi del GSUN/SIN, divulgare la metodologia dell'esame e il potenziale ruolo a cui la metodica. giustamente aspira.

Dall'alto della Sua brillante carriera, cosa consiglia, specialmente in questo periodo di grandi cambiamenti della sanità pubblica e della nefrologia in particolare, ai giovani nefrologi?

Credo si possa prevedere un futuro di grande impegno per il nefrologo abilitato all'esercizio delle metodiche ultrasonografiche.

- Il giovane Collega deve prendere sempre più confidenza non solo con gli aspetti diagnostici ma, soprattutto, con quelli interventistici e con le nuove tecnologie; mi riferisco in modo particolare ai mezzi di contrasto di II generazione e all'imaging parametrico.

- $\quad$ Nel caso in cui la proposta di dotare tutti i Medici di Medicina Generale di un ecografo portatile trovasse applicazione, questi dovranno interfacciarsi anche con il nefrologo, e il nostro gruppo dovrà abilmente inserirsi in questo filone.

- Tra qualche anno tutti i nefrologi dovranno avere nello studio in cui effettuano visite (pubbliche o private) un ecografo (così come il cardiologo dispone di un elettrocardiografo).

- Il terzo aspetto è quello della ricerca e delle pubblicazioni. Ricordo che, quando il Prof. F.P. Schena, in una riunione di tutti i Gruppi di Studio, chiese un impegno in questa direzione, molti si preoccuparono; oggi basta sfogliare le nostre riviste di settore per vedere come questo impegno sia stato rispettato oltre misura.

- $\quad$ Si può crescere soltanto conoscendosi e creando contatti e sinergie, per cui vedo con molto interesse un rafforzamento dei contatti con SIRM, SIUMB, SIEUN, SIC e, soprattutto, con la Società Italiana Medici di Medicina Generale.

L'attuale Consiglio Direttivo del Gruppo di Studio ha proposto alla SIN di cambiare nome, cioè di passare da Gruppo di Studio di Ecotomografia Renale a Gruppo di Studio di Ultrasonologia in Nefrologia. Come vede questo aggiornamento?

Era ora che il GSER si evolvesse, nel senso che la figura del nefrologo ecografista non si occupa più di semplice ecografia $B$-mode, come accadeva negli anni '90, ma di Doppler, ecocontrastografia e così via, e che il campo d'azione del nefrologo non è più e non può essere solo il rene ma, sostanzialmente, è di tipo internistico/interventistico.

Con quest'ultima domanda, caro Prof. Buccianti, La ringrazio per aver scritto questa pagina di storia del nostro gruppo e per tutto quello che, insieme ai "fantastici" quattro, ha fatto e ci ha lasciato in eredità.

\section{Disclosures}

Financial support: No financial support was received for this submission. Conflict of interest: The author has no conflict of interest. 